\title{
Optical Fibres for Distributed Corrosion Sensing - Architecture and Characterisation
}

\author{
Roman Kostecki ${ }^{1, a}$, Heike Ebendorff-Heidepriem ${ }^{1}$, Stephen C. Warren-Smith ${ }^{1}$, \\ Grant McAdam², Claire Davis ${ }^{2}$ and Tanya M. Monro ${ }^{1}$ \\ ${ }^{1}$ Institute for Photonics \& Advanced Sensing and School of Chemistry \& Physics, \\ University of Adelaide, Adelaide, SA 5005, Australia \\ ${ }^{2}$ Defence Science and Technology Organisation, Fishermans Bend, Victoria, Australia \\ aroman.kostecki@adelaide.edu.au
}

Keywords: Corrosion, Sensing, Microstructured optical fibres

\begin{abstract}
This paper summarises recent work conducted on the development of exposed core microstructured optical fibres for distributed corrosion sensing. Most recently, exposed-core fibres have been fabricated in silica glass, which is known to be reliable under a range of processing and service environments. We characterise the stability of these new silica fibres when exposed to some typical sensing and storage environments. We show the background loss to be the best achieved to date for exposed-core fibres, while the transmission properties are up to $\sim 2$ orders of magnitude better than for the previously reported exposed-core fibres produced in soft glass. This provides a more robust fibre platform for corrosion sensing conditions and opens up new opportunities for distributed optical fibre sensors requiring long-term application in harsh environments.
\end{abstract}

\section{Introduction}

The high acquisition costs associated with modern military and civilian aircraft coupled with tighter budgets has resulted in the need for greater utilisation of existing aircraft fleets. Typically, military aircraft have a planned life-of-type of 25-30 years. In Australia, in many cases, almost twice this time is achieved before the aircraft is retired [1]. For civilian aircraft flying today, approximately $20 \%$ are considered to be aging, and as that number increases so does the need for heightened fleet monitoring by airlines and manufacturers [2] and continued intense focus and surveillance of these activities by regulatory agencies [3].

With advancing aircraft age there is an increased possibility that protective coatings will break down or be damaged, resulting in exposure of the base material to the environment and an increased possibility of corrosion [2]. The continual cycling of the structure also increases the possibility of structural fatigue damage, which constitutes one of the most significant safety concerns due to the potential for corrosion to combine with structural fatigue [4]. While attempts are made to prevent and reduce the effect of corrosion, in practice it is impossible to completely avoid. It is therefore essential that corrosion processes are monitored so that repair time and costs are minimised, and the structure is maintained in a safe operating condition.

Corrosion is most difficult to detect in the hidden metallic structure of an aircraft, such as lap joints, stiffeners and under sealant beads, since access to these areas usually requires time consuming and therefore costly disassembly [1]. Such areas are recognised as being particularly prone to corrosion, due to the possibility of creating a micro-environment within the crevice between the plates, accelerating the corrosion process [4]. Current non-destructive inspection methods, typically based on the use of a hand held ultrasonic probe, require a 5-10\% section loss for corrosion to be reliably detected, at which stage the damage may be too severe to repair, and so the component often needs to be replaced [1]. Clearly a more proactive approach is needed to detect the initial onset of corrosion within these difficult to inspect areas.

Detecting corrosion in these areas is a distributed problem, and therefore requires a detection and/or monitoring system with the potential for distributed measurements, alerting the operator to the onset of corrosion. To monitor corrosion requires a sensor capable of measuring the changes that occur as 
a result of the corrosion process. Many of the aircraft fuselage components are fabricated from aluminium-based alloys, hence aluminium (Al) ions are of particular interest because they are not only an indicator of the onset of corrosion [1], but also have the potential to be an environmental hazard [5].

Optical fibre based sensors are inherently suited to distributed sensing [6] and are typically in the order of only a few hundred microns in diameter making them very lightweight and suitable for embedment in inaccessible corrosion-prone areas. In addition they can be functionalised to detect particular species of interest [1] such as Aluminium.

\section{Principles and Background}

The Australian Defence Science and Technology Organisation (DSTO) have been collaborating for several years with The University of Adelaide towards the development of a distributed fibre optic detection and monitoring system, capable of identifying corrosion in particularly prone areas of an aircraft fuselage [6]. The collaboration originated under the Centre of Expertise in Photonics (CoEP), and is now incorporated within the Institute for Photonics \& Advanced Sensing (IPAS). Ultimately, the optical fibre is intended to be placed in situ and be capable of detecting the onset and monitor the progress of corrosion anywhere along its length within the aircraft fuselage.

Conventional Optical Fibres. Initial work on the fluorometric detection of Al cations by the DSTO in conjunction with Monash University focussed on the use of conventional large core multimode fibres [1]. A range of different permeable polymer systems, doped with a compound that fluoresces in the presence of $\mathrm{Al}$ cations, were evaluated. The most promising of these was a flexible polyurethane the main constituent of which is a polyether polymer, poly(oxyethylene-cooxypropylene), known as 3-PEG. This is a random copolymer of polypropylene oxide and polyethylene oxide with a mole ratio of 1:3. The average molecular weight is of the order of $5000 \mathrm{~g} \cdot \mathrm{mol}^{-1}$, and is a cross-linked resin with a stoichiometric amount of 1,6-diisocyanatohexane (also known as hexamethylene diisocyanate, HDI) to produce a soft rubbery like polyurethane referred to as 3-PEG/PU. The 3-PEG/PU can be doped with a fluorescent compound, 8-hydroxyquinoline (8-HQ), which reacts with Al cations form a rigid octahedral complex which fluoresces at $516 \mathrm{~nm}$ under UV excitation at 360-390 $\mathrm{nm}$. The reaction chemistry is shown in Figure 1.

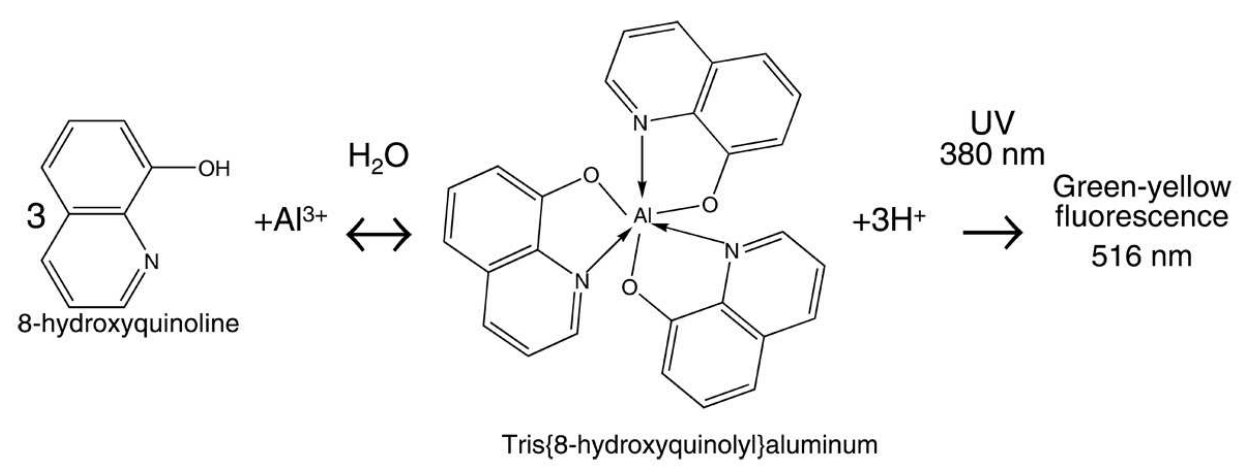

Figure 1. Reaction sequence for 8-hydroxyquinoline with Al cations [1].

The doped 3-PEG/PU was dip-coated onto the distal end of a conventional wide core optical fibre to create a sensing polymer bead at the fibre tip. This bead was exposed to 8-HQ in solution to allow the dopant to diffuse into the polymer, and then allowed to dry naturally prior to placement into the channel of a simulated lap joint in a region where the corrosion protective coating had been removed. This lap joint was then placed in a salt spray chamber for a period of 14 days to accelerate the corrosion process, as shown in Figure 2. 


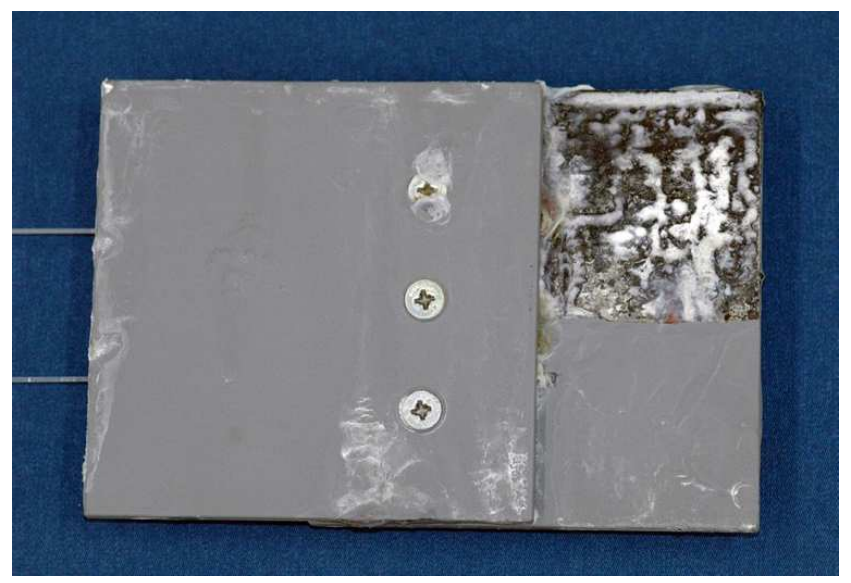

Figure 2. Simulated lap-joint after exposure in salt spray chamber for 2 weeks.

The sensing fibre was excited using an UV LED excitation source and the back-reflected fluorescence measured via a $3 \mathrm{~dB}$ coupler using a micro spectrophotometer (Ocean optics). A schematic diagram of the experimental set-up is shown in Figure 3(a). The measured back fluorescence as a function of exposure time in the salt-spray chamber is plotted in Figure 3(b).

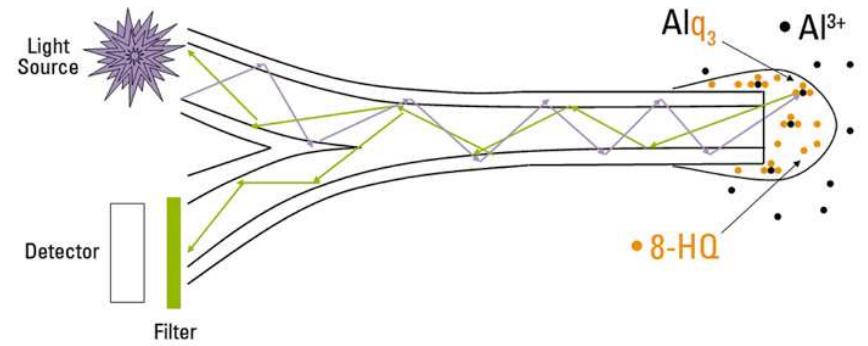

(a)

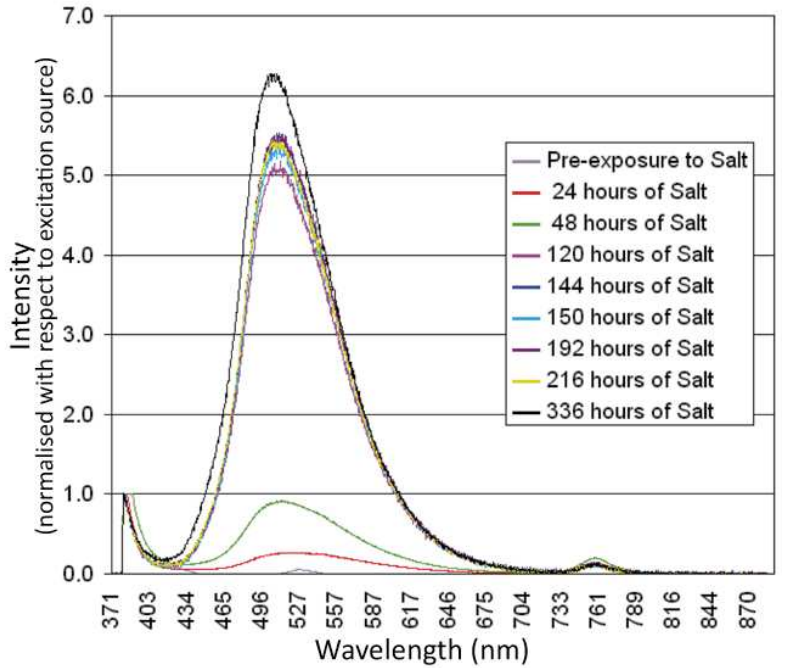

(b)

Figure 3. (a) Schematic for point detection from a doped polymer bead, and (b) the measured back reflected fluorescence from the fibre tip as a function of exposure time in the salt spray chamber.

For this case, where the bead is attached to the end of an optical fibre, there are fewer physical constraints on the properties of the permeable coating used to contain the fluorescent species compared to a distributed sensor. Ideally, it would be preferable to create sensitivity to Al cations by coating the fibre along the length, thus creating a distributed sensor rather than a local (point) sensor. In order to make distributed measurements the fraction of guided light propagating along the outside of the core needs to be accessed, which requires careful tailoring of the cladding refractive index to optimise the light/fluorophore interaction.

The concept of distributed detection of fluorescence from 8-HQ complex with Al cations in solution was demonstrated at Swinburne University in conjunction with the DSTO [7]. By using a large solid core $(200 \mu \mathrm{m})$ silica fibre with the cladding removed, it was shown that Optical Time Domain Reflectometry (OTDR) could be used to not only detect the presence of the Al cations but also locate the position of this fluorescence $80 \mathrm{~m}$ along the fibres length. Figure 4(a) shows the setup, where photon counts were accumulated by the time-gated electronics in bins of $2 \mathrm{~ns}$ width, which corresponds to a length resolution along the fibre of $0.2 \mathrm{~m}$, assuming a propagation velocity of 
$2 \times 10^{8} \mathrm{~m} \cdot \mathrm{s}^{-1}$. An accumulation time of $10 \mathrm{~min}$ was used to obtain adequate signal-to-noise discrimination. These photon bin accumulations corresponded to the return time of flight of the fluorescence that originate at a distance along the fibre. The signal detected is shown in Figure 4(b), which represents the time domain response of the detection system when the de-clad fibre section was immersed in a $5 \times 10^{-3} \mathrm{M}$ solution of the 8 -HQ complex.

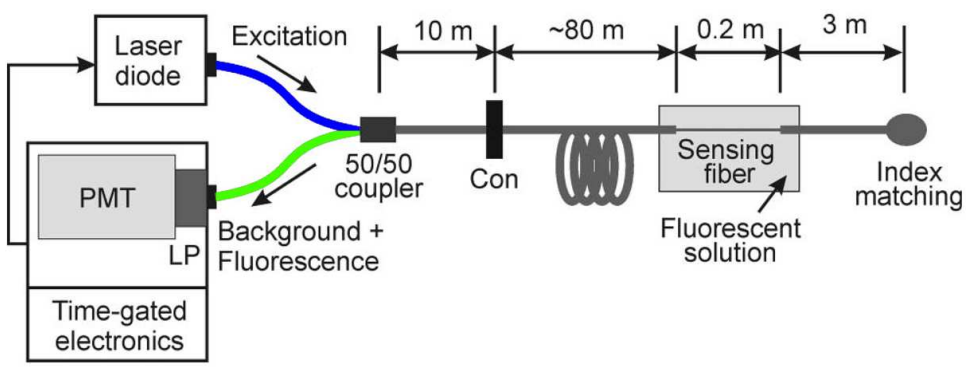

(a)

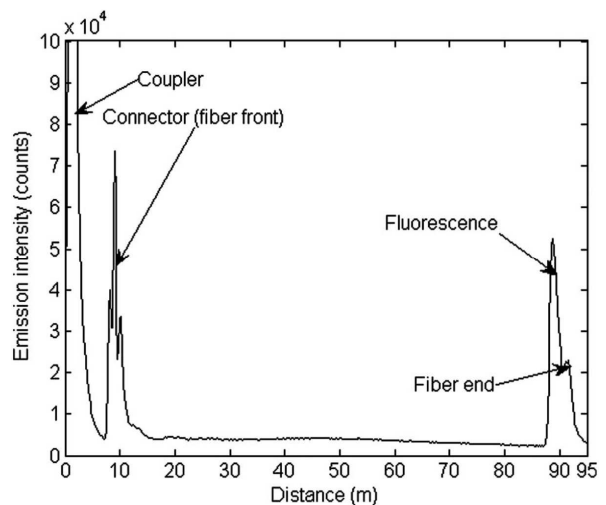

(b)

Figure 4. (a) OTDR setup for distributed measurements of Al cations (LP, long pass; Con, connector) in solution using a silica fibre with $200 \mu \mathrm{m}$ core; with which the (b) time of flight resolved fluorescence response of the 8-HQ complex within solution corresponds to detection position along the fibre [7].

This OTDR experiment, conducted at Swinburne University, and described in detail in Ref. [7], shows that distributed fluorescence detection of the Al cations is possible. The fraction of power from the guided light propagating along the outside of the core was able to be used to excite the 8HQ complex and the fraction of emitted florescence recaptured by the fibre core could be measured by the time-gated electronics. However, using such a large core diameter $(200 \mu \mathrm{m})$ less than $0.1 \%$ of the total optical power propagates outside the core, making it impractical for applications requiring low detection limits [8]. Nevertheless, it is possible to increase this power fraction by reducing the core diameter.

Figure 5 shows a theoretical estimate [9] of the power fraction propagating outside a circular silica core, for core diameters from $460 \mathrm{~nm}$ to $10 \mu \mathrm{m}$. The excitation and fluorescence wavelengths used for this study were $375 \mathrm{~nm}$ and $516 \mathrm{~nm}$ respectively, being the excitation and fluorescence wavelengths of the 8-HQ complex, and for the region surrounding the silica core a refractive index of $\mathrm{n}=1.33$ was used. As a first approximation, the coupled incident beam waist was set at the same diameter as the core, which is reasonable for micron scale $(>1 \mu \mathrm{m})$ core diameters. The total fraction of guided light power (PF) that propagates outside the core, of all propagating modes excited by the incident beam as a function of the core diameter, is shown by the red curve in Figure 5(a). This PF curve shows that for core diameters less than $4 \mu \mathrm{m}$ the fraction of power available for fluorophore excitation starts to significantly increase, being 0.3 and $2.1 \%$ at 4 and $2 \mu \mathrm{m}$ diameters respectively. For core sizes below $1 \mu \mathrm{m}$ in diameter there is another significant PF increase, where the calculated values are 2.8 and $17 \%$ at $1 \mu \mathrm{m}$ and $460 \mathrm{~nm}$ diameters respectively. The corresponding fluorescence capture fraction (FCF) is shown by the blue curve in Figure 5(a). The FCF is defined as the fraction of fluorescent photons that are coupled to the guided mode(s) of the fibre. Although the total PF was used to calculate the FCF, for simplicity only the FCF into the fundamental mode $\left(\mathrm{HE}_{11}\right)$ of the fibre was considered for the special case of an attenuation-free fibre of infinite length. This FCF curve varies from a minimum of $1.1 \%$ to a maximum of $1.7 \%$. 


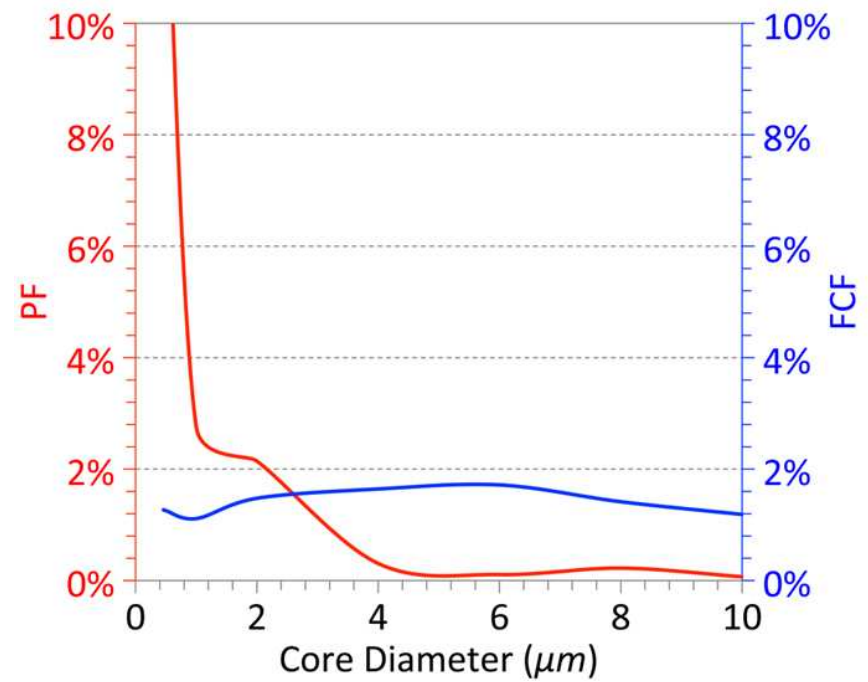

(a)

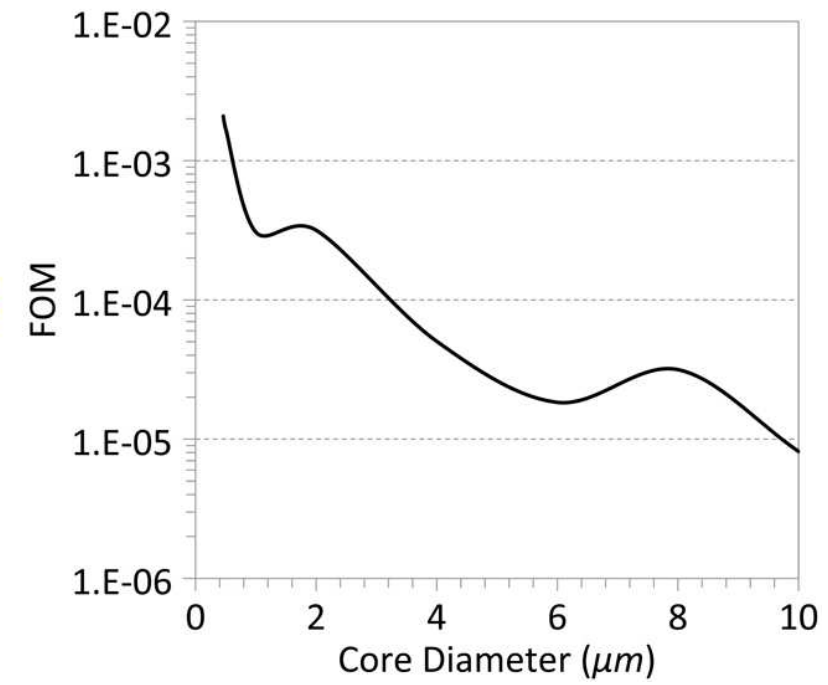

(b)

Figure 5. (a) [Red] Fraction of guided light power propagating outside a silica core as a function of core diameter assuming Gaussian beam excitation, and [Blue] the corresponding fluorescence capture fraction into the fundamental mode; and (b) the figure of merit as a combination of both.

The intensity of fluorescence that is measured from the backward propagating modes of the fibre is the result of both the incident excitation power and the number of fluorescence photons recaptured. These quantities can be brought together to provide a better understanding of the overall performance of the sensor as a function of the core diameter by defining a figure of merit, $\mathrm{FOM}=(\mathrm{PF})(\mathrm{FCF})[8]$. This FOM is shown in Figure 5(b), where we observe an order of magnitude increase in sensor performance between the peaks at 8 and $2 \mu \mathrm{m}$, and no significant change in performance from 2 to $1 \mu \mathrm{m}$. Another factor, which will influence the FOM, is the fibre loss, which increases with smaller core diameters (and therefore greater PF) due to the increased scattering resulting from surface imperfections. Previous work [8] has included the effect of fibre loss on the total FOM, and the next step is to include this with respect to this work.

Microstructured optical fibres (MOFs). Unlike glass nano-wires [10], a MOF [11] provides a means for obtaining long lengths of uniform micrometre-nanometre scale suspended and protected cores. A protected core geometry is necessary for sensing in harsh environments such as the application under consideration in this paper. In particular, the suspended-core fibre [12] design, which consists of a small glass core suspended by thin struts inside voids within the fibre, have found extensive use in chemical sensing applications [8, 13]. For these fibres the PF overlaps with the voids, and by controlling the size of the core, this overlap between the light and the voids can be adjusted. If the voids of the fibre are then filled with liquids or gases this controllable interaction allows sensing measurements to be performed, using methods such as direct absorption [14] or various fluorescent techniques [8, 15]. These suspended-core fibres dramatically improve fluorescence-based fibre sensors by making use of the increased PF provided by the micrometre scale core size and therefore increased sensor performance. Figure 6 shows a suspended-core fibre, recently fabricated within IPAS at the University of Adelaide, based on undoped high purity fused silica known as Suprasil F300HQ (Heraeus Quarzglas GmbH \& Co.KG).

Figure 6(a) shows the overall suspended-core structure, with the polymer coating removed, this example has an outside diameter of $270 \mu \mathrm{m}$, although smaller sizes can easily be achieved. An enlargement of the core and holes region in the centre of the fibre is shown in Figure 6(b), where the core of the fibre is the small triangular element in the centre of the image (highlighted by the green box), suspended on three thin struts. The three black voids are the holes within the fibre cross-section, which form the cladding region used to provide the refractive index contrast needed for light confinement, and can also be used as sample chambers, with each hole diameter being 12.4 $\mu \mathrm{m}$, defined as the diameter of a circle whose area is equal to the cross sectional area of the hole. 
The web thicknesses are at least $0.16 \mu \mathrm{m}$, while the core has an effective diameter of $1.7 \mu \mathrm{m}$, defined as the diameter of a circle whose area is equal to a triangle that fits wholly within the core area $[16]$.
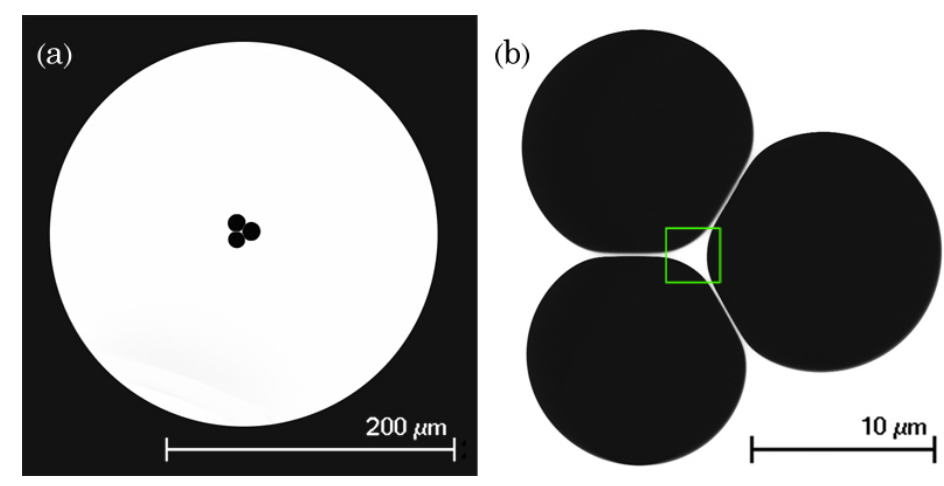

Figure 6. Scanning electron microscope cross section images of (a) the silica suspended-core fibre with a outside diameter of $270 \mu \mathrm{m}$; and, (b) an enlarged image of the core and hole region, where the core is highlighted by the green box, having an effective diameter of $1.7 \mu \mathrm{m}$.

While suspended-core fibres have provided a highly sensitive sensing platform [8, 14, 17-20], exploiting the significant fraction of guided power located within the holes, the closed structure makes it impossible to use them for distributed sensing applications. To overcome this, fabrication techniques that expose the core have been demonstrated by micro-machining fluidic side-channels at several locations along the fibre length [21-24], which results in short exposed regions in the order of tens of microns. This provides access to the core by the analyte, making it useful for real time sensing applications, but is still not practical for distributed sensing applications where long lengths are required. To overcome this problem, it has been demonstrated $[25,26]$ that effectively 'opening up' one of the voids within the fibre during the initial fabrication stages, creating an exposed-core fibre, can allow the guided light to interact with the surrounding medium anywhere along its length. This method provides the ability to fabricate exposed-core fibres hundreds of metres long with a fixed microstructured cross section.

The fabrication of exposed-core fibres is challenging due to their asymmetric cross section, where the method involved modification of the established cane and sleeve process [16] by introducing a wedge in the preform fabrication process [26]. These fibres were initially produced in soft glass (Schott F2) material, which has a glass transition temperature low enough to make it practical for extruding the structured preform [27]. The process, summarised in Figure 7, involved extruding an F2 soft glass billet into a wagon-wheel (WW) structured preform at high temperature and pressure. The WW preform was then drawn to a cane $(\sim 1 \mathrm{~mm}$ diameter $)$, using a fibre drawing tower, then inserted into an extruded F2 soft glass wedged jacket (sleeve). The cane-in-jacket preform was then drawn to fibre with an outer diameter of $\sim 160 \mu \mathrm{m}$ diameter (top-right of Figure 7 ) where the $\sim 3 \mu \mathrm{m}$ effective diameter core, in the exposed region highlighted by the green box, is suspended by three thin struts [26]. An enlarged view of the exposed core structured region of the fibre is shown in the bottom-right view of Figure 7. 


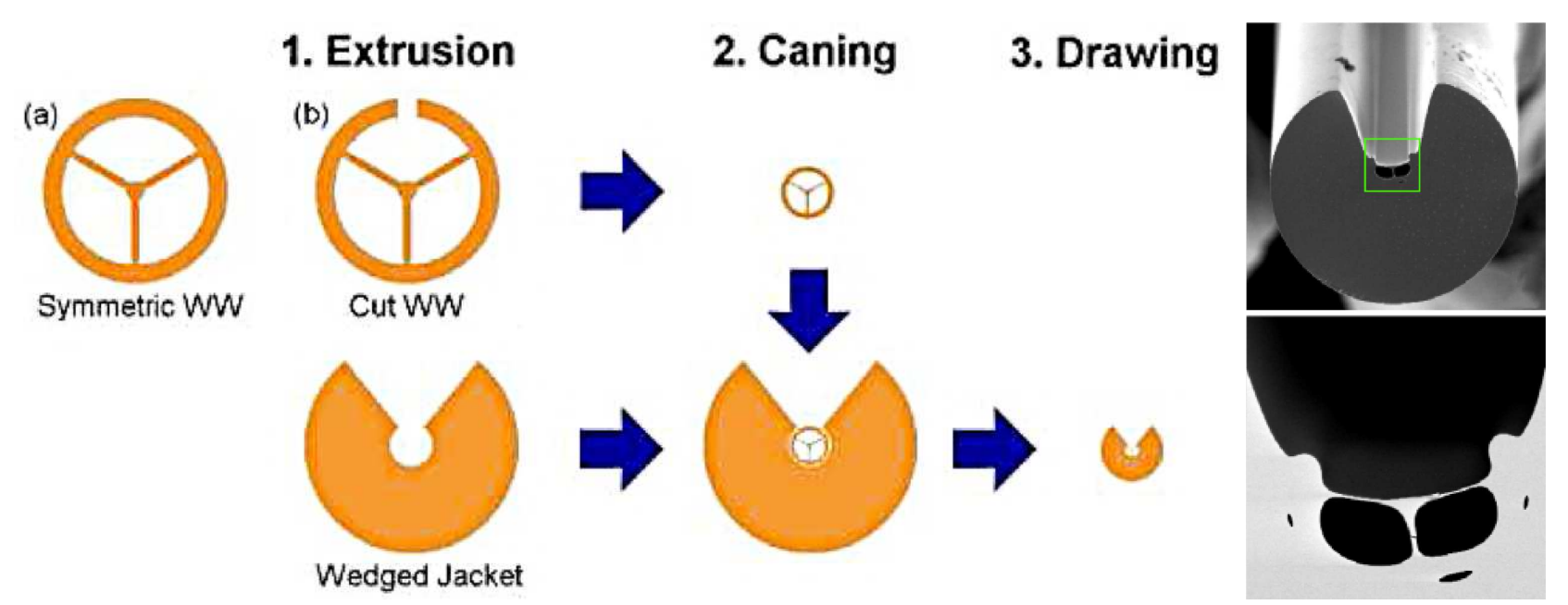

Figure 7. (Left) Schematic diagram of F2 soft glass exposed-core fibre fabrication process, with scanning electron microscope images of (top-right) the resulting exposed-core fibre with a outside diameter of $\sim 160 \mu \mathrm{m}$; with an enlargement of the area highlighted by the green box (bottom-right) showing the core, hole and strut structured region, where the core has an effective diameter of $\sim 3.0$ $\mu \mathrm{m}[26]$.

Fabrication of these exposed small-core fibres was a significant milestone in the development of the distributed fibre optic corrosion sensor. For the first time, small core distributed fluorescence measurements became possible [6], where the increased fraction of power from the guided light propagating outside the small core could be accessed anywhere along its length, whilst still being protected by the outside sleeve. Since the F2 soft glass is not transparent at UV wavelengths, another fluorophore (lumogallion) with excitation and fluorescence wavelengths at 502 and $588 \mathrm{~nm}$ respectively, was used for Al cation detection. Unfortunately, it was found that F2 soft glass was not suitable for long term use and/or harsh environments [26, 28], where the fibre loss increased by $0.4 \pm 0.048 \mathrm{dBm}^{-1}$ day $^{-1}$ while in storage. These loss and deterioration issues associated with the soft glass material from which they were fabricated precluded their long-term practical use under harsh conditions.

To produce a distributed fibre optic corrosion sensor capable of withstanding the intended harsh conditions, other fibre materials need to be investigated. Silica is known to be reliable under a range of processing and use environments, with relatively better mechanical and thermal stability [29]. Highly homogeneous, high purity bulk material is commercially available, which has led to silica telecommunications fibres regularly being made with low loss $(\sim 0.2 \mathrm{~dB} / \mathrm{km}$ at NIR wavelengths $)$ [30]. Silica also has high transmission in the UV-Vis-NIR spectral range, making it suitable for a greater range of fluorophores, including 8-HQ. In the next section we report the first silica exposedcore fibres produced, and characterise their environmental stability when exposed to typical sensing and storage environments.

\section{Silica Exposed-Core Fibres}

As discussed in the previous section, the rationale behind producing silica exposed-core fibres for corrosion sensing applications was to make a physically robust sensing element for long length distributed sensing applications. The fabrication of a microstructured fibre with the core exposed along the whole length provides near-instantaneous measurements of fluorescence intensity while additionally allowing the opportunity for spatial measurements along the length of the fibre through temporal detection methods such as OTDR [26]. High purity fused silica Suprasil F300HQ was chosen because of its low hydroxide $(\mathrm{OH})$ content and availability with tight geometric tolerances [32]. This material has high transmission in the UV-Vis-NIR spectral range, making it suitable for a range of fluorophores, and is known to be reliable under a range of processing and use environments, with good mechanical and thermal stability [29]. Unlike soft glass, the glass transition temperature of silica is high making it challenging to extrude, and since the exposed-core fibres are asymmetric, new fabrication methods needed to be established. 
Fabrication. The silica exposed-core fibre preform, as shown in Figure 8(a), was fabricated by drilling three holes along the length of the fused silica rod, with centres spaced such that they form an equilateral triangle, and a slot was cut along the length of one of the holes. These methods expand on a combination of work previously shown by Webb et al. [14] for fabricating silica suspended-core fibres by drilling the preform, and Warren-Smith et al. [26] for cutting a thin slot into the side of the symmetric preform (soft glass) in order to expose the core region. The preform was cleaned after drilling using acetone and ultrapure (Milli-Q ${ }^{\mathrm{TM}}$ ) water, then etched in a buffered hydrofluoric acid solution (BHF), made using 6 volumes of ammonium fluoride $\left(\mathrm{NH}_{4} \mathrm{~F}, 40 \%\right.$ solution) to 1 volume of hydrofluoric acid (HF, 50\% solution), with a known etch rate of 100-250 $\mathrm{nm} / \mathrm{min}[33]$.
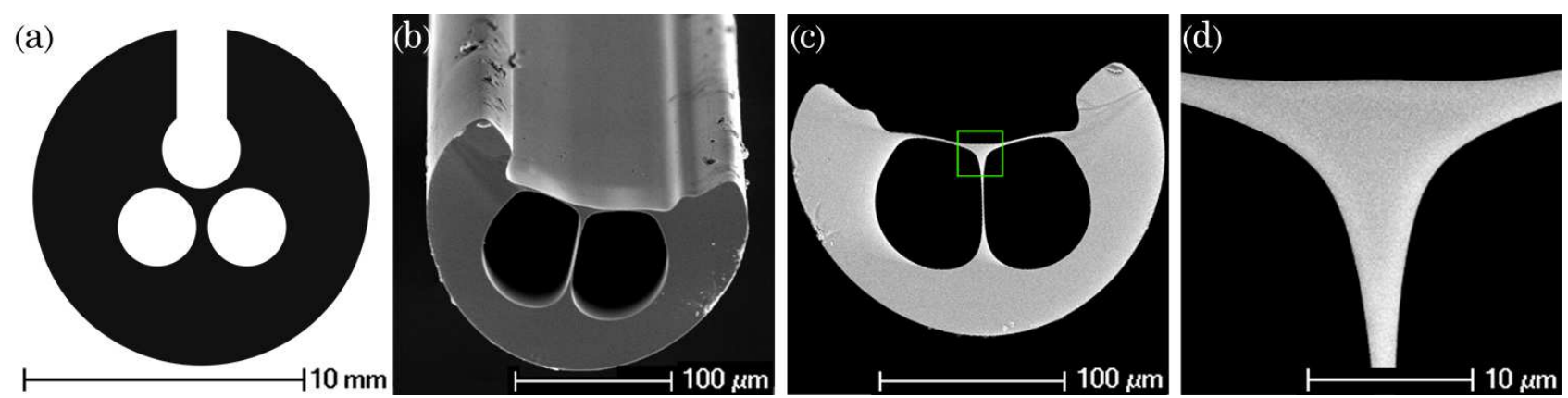

Figure 8. (a) Cross section of the preform fabricated from $\varnothing 12 \mathrm{~mm}$ F300HQ silica rod; and, scanning electron microscope images of (b) the silica exposed-core fibre with (c) the cross section measured at the maximum to be $\varnothing 202 \mu \mathrm{m}$; and, (d) an enlarged image of the core having an effective diameter of $10.0 \mu \mathrm{m}$ [31].

A single long length $(>100 \mathrm{~m})$ of silica exposed-core fibre, as shown in Figure 8(b), was drawn using a $6 \mathrm{~m}$ tower with a graphite furnace, positive pressurisation system and automated diameter control. The outside diameter and effective core diameter, as shown in the SEM images of Figures $8(\mathrm{c})-8(\mathrm{~d})$, were measured as 202 and $10 \mu \mathrm{m}$ respectively. The fibre loss measurement results taken directly after fibre drawing were $1.12 \pm 0.15,1.10 \pm 0.08$ and $1.43 \pm 0.39 \mathrm{dBm}^{-1}$ at 532,900 and 1550 $\mathrm{nm}$ respectively [31]. This relatively large core silica exposed-core fibre was useful to enable surface analysis of the core, and for determining the environmental stability when exposed to some typical sensing and storage environments. For sensing purposes, smaller cores have greater merit, as discussed in the previous section. To fabricate an exposed small-core fibre, a combination of the method used above with the caning and sleeving process, shown in Figure 7, was used. A single long length silica exposed-core fibre, as shown in Figure 9(a), was drawn where the cross section is shown in Figure 9(b) with an outside diameter of $150 \mu \mathrm{m}$. Figure 9(c) shows the hole and strut structure which forms the triangular shaped core exposed to the outside environment on one side. The core in this case, shown in Figure 8(d), has an effective diameter of $1.7 \mu \mathrm{m}$.
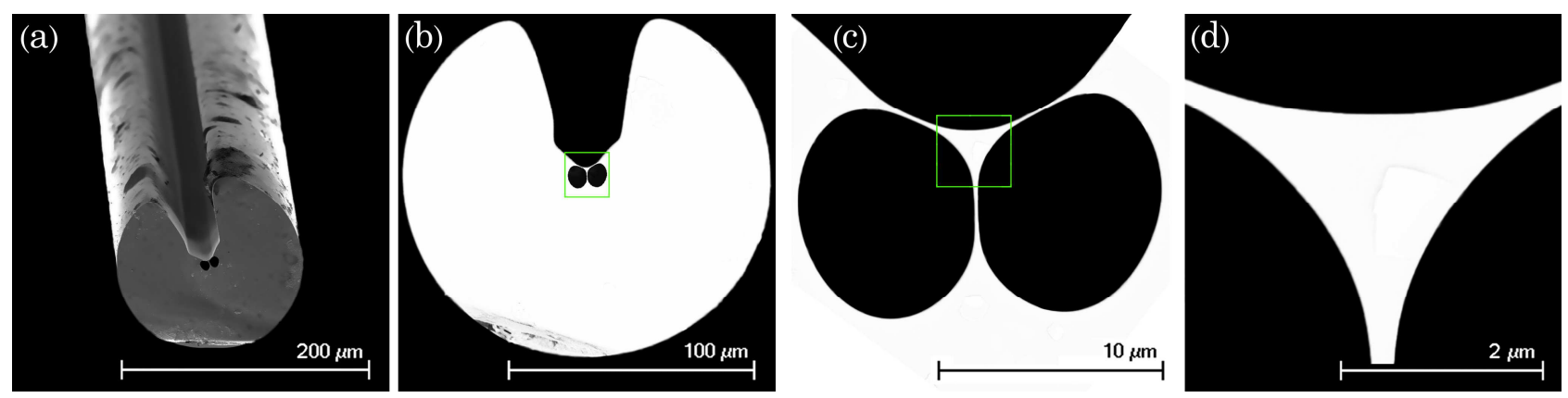

Figure 9. Scanning electron microscope images of (a) the small core silica exposed-core fibre with (b) the cross section measured at the maximum to be $\varnothing 150 \mu \mathrm{m}$; (c) the structured core, holes and strut region; and, (d) an enlarged image of the core having an effective diameter of $1.7 \mu \mathrm{m}$. 
Environmental Stability. The silica exposed-core fibre, shown in Figure 8, was used for measuring the deterioration characteristics in transmission properties when exposed to typical sensing and processing environments, where the structure also provides access to the core for post exposure surface analysis. In the case of corrosion detection, it is expected that the fibre might be exposed to the environment, coming in contact with the surrounding air or water.

To measure the exposure induced deterioration in the transmission properties of the silica exposedcore fibre, any changes over time in the transmission characteristics were fitted to the equation,

$$
\mathrm{P}_{\lambda, \mathrm{t}}=\mathrm{P}_{\lambda, 0} 10^{-\xi \mathrm{t} t / 10}
$$

where $\xi$ is the loss change in $\mathrm{dB} / \mathrm{day}$.

The result of the change in fibre loss ( $\xi$ in Eq. (1)) for a $4.2 \mathrm{~m}$ length of the exposed-core fibre exposed to air for $180 \mathrm{hrs}$ is shown in Figure 10(a), where the 95\% confidence interval is also shown in black. This result shows that there is a sharp loss peak at $515 \mathrm{~nm}$, equivalent to 0.043 $\mathrm{dBm}^{-1} \mathrm{day}^{-1}$, and a broad loss from 450 to $900 \mathrm{~nm}$ with a peak of $0.023 \mathrm{dBm}^{-1} \mathrm{day}^{-1}$. At wavelengths from 900 to $1340 \mathrm{~nm}$ the loss is at $0.0043 \mathrm{dBm}^{-1} \mathrm{day}^{-1}$, and drops below the detection limit of the experiment for wavelengths $>1340 \mathrm{~nm}$. This result shows that the air exposure induced deterioration in the transmission properties of the silica exposed-core fibre is $\sim 2$ orders of magnitude better than for the exposed-core fibres produced in F2 soft glass. This measurement was repeated for a $1 \mathrm{~m}$ length of the silica exposed-core fibre with an $8 \mathrm{~cm}$ centrally located section of the fibre submersed in Milli-Q water. Figure 10(b) shows that the reduction in the transmission properties of the fibre is $0.067 \mathrm{dBm}^{-1}$ day $^{-1}$ for wavelengths shorter than $1450 \mathrm{~nm}$.
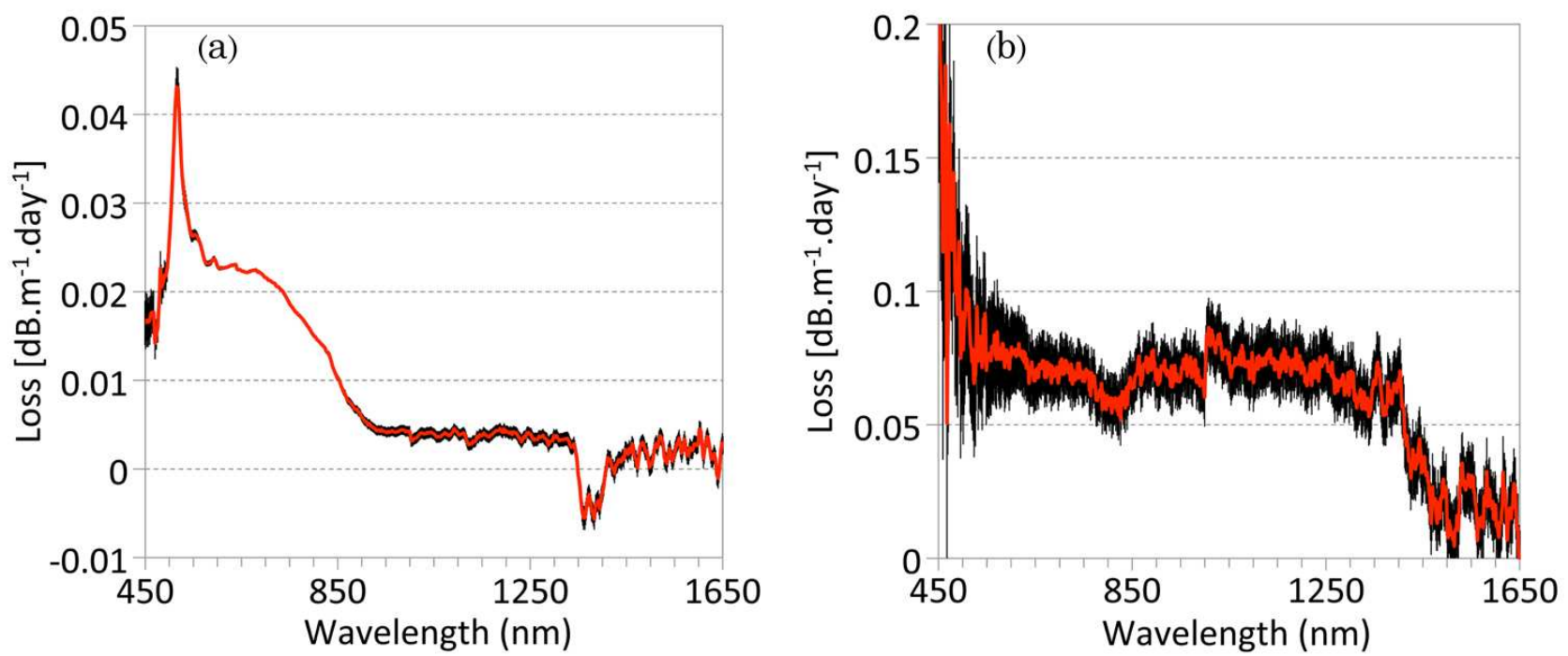

Figure 10. Deterioration in the transmission properties of the silica exposed-core fibre when exposed to (a) air; and, (b) water. The red line shows the change in fibre loss ( $\xi$ in Eq. (1)) with the $95 \%$ confidence interval shown in black.

This deterioration in the transmission properties is expected to come from changes in the mechanical and/or compositional characteristics at the core surface, causing light scattering effects $[34,35]$. When the core diameter is reduced, these light scattering effects are expected to increase, as a greater portion of guided light travels outside the core [19]. Further work is required to quantitatively determine the effect that core size has on the deterioration in the transmission properties of the silica exposed-core fibre.

In order to determine the differences between the mechanical and compositional characteristics of the exposed sample surfaces, nanometre-scale topographical and phase mapping of the exposedcore fibre core surfaces was performed using Tapping Mode Atomic Force Microscopy (AFM). Figures 11(a)-11(c) and 11(d)-11(f) show the AFM phase and topology results of a $25 \mu^{2}$ section across the core for the exposed-core fibre exposed to air for 19 days and Milli-Q water for 3 days 
respectively. For the core area exposed to air, the nanometre scale spikes in the topology and phase shift suggest small impurities within the surface structure, while the bulk of the material is homogeneous in composition with nanometre scale roughness. For the core exposed to water, large peaks measuring $>100 \mathrm{~nm}$ in height and several microns across the surface suggest that impurities from the water have been deposited onto the core. The darkest areas in the phase image show up on the topology to be slightly lower than the surrounding bulk, which may also be an indication of surface damage such as micro-fracturing [34].
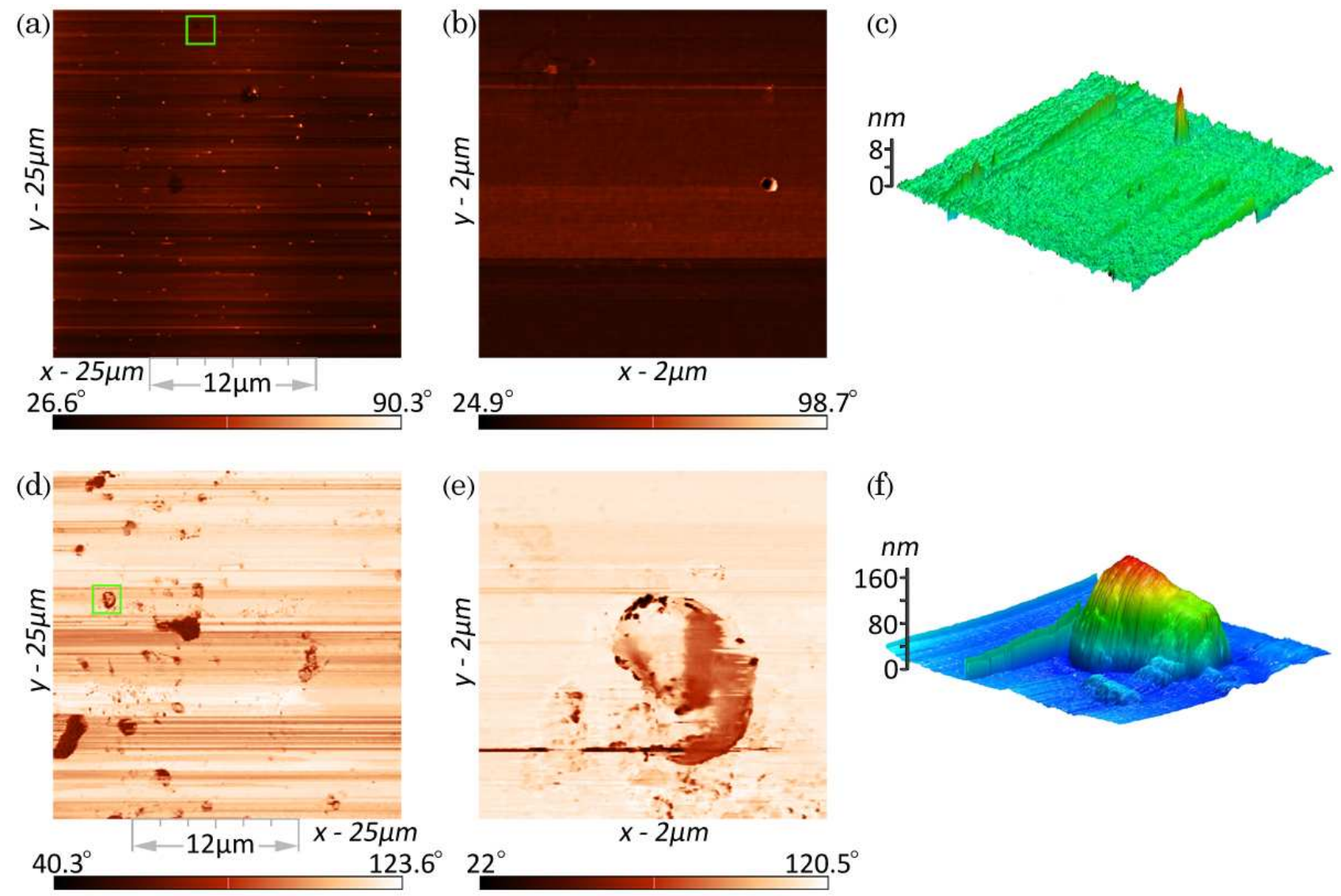

(f)

Figure 11. Tapping Mode Atomic Force Microscopy images of the exposed-core fibres exposed to (a)-(c) air, and (d)-(f) water. (a) and (d) show the phase images across the core region indicated by the $12 \mu \mathrm{m}$ area on the x-axis, with [(b) and (e) respectively] enlarged phase images of the area shown by the green box; and, (c) and (f) showing their respective topologies.

\section{Conclusion}

We have summarised recent work done to develop a distributed fibre optic detection and monitoring system, capable of identifying corrosion in particularly prone areas of an aircraft fuselage. This research led to an exposed-core fibre distributed corrosion sensing element produced in lead silicate soft glass, which was found to deteriorate quickly when exposed to the harsh conditions of a simulated corrosive environment, making it impractical for long term use on Defence Platforms. Further work has been presented on newly developed exposed-core fibres fabricated using silica material, which is known to be reliable under a range of processing and service environments. These new silica exposed-core fibres show a relatively low loss with deterioration in the transmission properties being $\sim 2$ orders of magnitude better than that for the soft glass version. Although the silica material shows good stability in air and water, the build-up of contaminates on the surface and micro-fracturing are the most likely cause for the degree of deterioration measured. In the future, further practical issues need to be solved, particularly in how to package the exposedcore fibre such that it is sensitive to Al cations but protected from the harsh environment of an aircrafts fuselage. Further work is also needed to determine if the degree of deterioration measured is sufficiently low for practical application to the intended use on Defence platforms. 


\section{Acknowledgments}

The authors acknowledge Peter Henry for his contribution to the silica fibre drawing, and the Australian Defence Science and Technology Organisation (under the Signatures, Materials and Energy Corporate Enabling Research Program) for support of the suspended core Si fibre development. T. Monro acknowledges the support of an Australian Research Council Federation Fellowship.

\section{References}

[1] G. McAdam, P. J. Newman, I. McKenzie, C. Davis, and B. R. W. Hinton, Fiber optic sensors for detection of corrosion within aircraft, Struct. Health Monit. 4 (2005) 47-56.

[2] J. Hall, Corrosion prevention and control programs for Boeing airplanes, SAE Technical Paper 931259 (1993)

[3] Civil Aviation Safety Regulations 1998, Part 39.001(1), AD/B737/52 Amdt 3 - Corrosion Prevention and Control Program (CPCP) (2011)

[4] I. McKenzie and P. J. Newman, Methods for detection of corrosion in aircraft structures using fiber optic technology, In: Tri-Service Corrosion Conference (1999) 15-19 November, Myrtle Beach, South Carolina.

[5] J. Scancar and R. Milacic, Aluminium speciation in environmental samples: a review, Anal. Bioanal. Chem. 386 (2006) 999-1012.

[6] S. Warren-Smith, E. Sinchenko, P. Stoddart, and T. Monro, Distributed fluorescence sensing using exposed core microstructured optical fiber, IEEE Photon. Technol. Lett. 22 (2010) $1385-1387$.

[7] E. Sinchenko, W. E. K. Gibbs, C. E. Davis, and P. R. Stoddart, Characterization of timeresolved fluorescence response measurements for distributed optical-fiber sensing, Appl. Opt. 49 (2010) 6385-6390.

[8] E. P. Schartner, H. Ebendorff-Heidepriem, S. C. Warren-Smith, R. T. White, and T. M. Monro, Driving down the detection limit in microstructured fiber-based chemical dip sensors, Sensors 11 (2011) 2961-2971.

[9] S. C. Warren-Smith, S. Afshar V., and T. M. Monro, Theoretical study of liquid-immersed exposed-core microstructured optical fibers for sensing, Opt. Express 16 (2008) 9034-9045.

[10] L. Tong, R. R. Gattass, J. B. Ashcom, S. He, J. Lou, M. Shen, I. Maxwell, and E. Mazur, Subwavelength-diameter silica wires for low-loss optical wave guiding, Nature 426 (2003) 816-819.

[11] T. M. Monro and H. Ebendorff-Heidepriem, Progress in microstructured optical fibers, Annu. Rev. Mater. Res. 36 (2006) 467-495.

[12] T. M. Monro, S. Warren-Smith, E. P. Schartner, A. Francois, S. Heng, H. EbendorffHeidepriem, and S. Afshar V., Sensing with suspended-core optical fibers, Opt. Fiber Technol. 16 (2010) 343-356.

[13] S. C. Warren-Smith, S. Heng, H. Ebendorff-Heidepriem, A. D. Abell, and T. M. Monro, Fluorescence-based aluminum ion sensing using a surface-functionalized microstructured optical fiber, Langmuir 27 (2011) 5680-5685.

[14] A. Webb, F. Poletti, D. Richardson, and J. Sahu, Suspended-core holey fiber for evanescentfield sensing, Opt. Eng. 46 (2007) 010503.

[15] T. M. Monro, S. Warren-Smith, E. P. Schartner, A. Francois, S. Heng, H. EbendorffHeidepriem, and S. Afshar V., Sensing with suspended-core optical fibers, Opt. Fiber Technol. 16 (2010) 343-356.

[16] H. Ebendorff-Heidepriem, S. C. Warren-Smith, and T. M. Monro, Suspended nanowires: fabrication, design and characterization of fibers with nanoscale cores, Opt. Express 17 (2009) $2646-2657$. 
[17] T. G. Euser, J. S. Y. Chen, M. Scharrer, P. S. J. Russell, N. J. Farrer, and P. J. Sadler, Quantitative broadband chemical sensing in air-suspended solid-core fibers, J. Appl. Phys. 103 (2008) 103108.

[18] A. Mazhorova, A. Markov, A. Ng, R. Chinnappan, O. Skorobogata, M. Zourob, and M. Skorobogatiy, Label-free bacteria detection using evanescent mode of a suspended core terahertz fiber, Opt. Express 20 (2012) 5344-5355.

[19] S. Afshar V., S. C. Warren-Smith, and T. M. Monro, Enhancement of fluorescence-based sensing using microstructured optical fibres, Opt. Express 15 (2007) 17891-17901.

[20] Y. Ruan, E. P. Schartner, H. Ebendorff-Heidepriem, P. Hoffmann, and T. M. Monro, Detection of quantum-dot labelled proteins using soft glass microstructured optical fibers, Opt. Express 15 (2007) 17819-17826.

[21] C. M. B. Cordeiro, C. J. S. de Matos, E. M. dos Santos, A. Bozolan, J. S. K. Ong, T. Facincani, G. Chesini, A. R. Vaz, and C. H. B. Cruz, Towards practical liquid and gas sensing with photonic crystal fibres: side access to the fibre microstructure and single-mode liquidcore fibre, Meas. Sci. Technol. 18 (2007) 3075-3081.

[22] C. Martelli, P. Olivero, J. Canning, N. Groothoff, B. Gibson, and S. Huntington, Micromachining structured optical fibers using focused ion beam milling, Opt. Lett. 32 (2007) $1575-1577$.

[23] A. van Brakel, C. Grivas, M. N. Petrovich, and D. J. Richardson, Micro-channels machined in microstructured optical fibers by femtosecond laser, Opt. Express 15 (2007) 8731-8736.

[24] J. P. Parry, B. C. Griffiths, N. Gayraud, E. D. McNaghten, A. M. Parkes, W. N. MacPherson, and D. P. Hand, Towards practical gas sensing with micro-structured fibres, Meas. Sci. Technol. 20 (2009) 075301.

[25] F. M. Cox, R. Lwin, M. C. J. Large, and C. M. B. Cordeiro, Opening up optical fibres, Opt. Express 15 (2007) 11843-11848.

[26] S. C. Warren-Smith, H.Ebendorff-Heidepriem, T. C. Foo, R. Moore, C. Davis, and T. M. Monro, Exposed-core microstructured optical fibers for real-time fluorescence sensing, Opt. Express 17 (2009) 18533-18542.

[27] H. Ebendorff-Heidepriem and T. M. Monro, Extrusion of complex preforms for microstructured optical fibers, Opt. Express 15 (2007) 15086-15092.

[28] S. C. Warren-Smith, H. Ebendorff-Heidepriem, S. Afshar V., G. McAdam, C. Davis, and T. Monro, Corrosion sensing of aluminium alloys using exposed-core microstructured optical fibres, Materials Forum 33 (2009) 110-121.

[29] K. Richardson, D. Krol, and K. Hirao, Glasses for photonic applications, Int. J. Appl. Glass Sci. 1 (2010) 74-86.

[30] M. Li and D. Nolan, Optical transmission fiber design evolution, J. Lightwave Technol. 26 (2008) 1079-1092.

[31] R. Kostecki, H. Ebendorff-Heidepriem, C. Davis, G. McAdam, S. C. Warren-Smith, and T. M. Monro, Silica exposed-core microstructured optical fibers, Opt. Mater. Express 2 (2012) $1538-1547$.

[32] Heraeus Quarzglas GmbH \& Co. KG, Pure Silica Rods for Specialty Fiber Applications, 1st ed. (2012), http://heraeus-quarzglas.com/.

[33] M. Y. Sim and S. Gleixner, Studying the etch rates and selectivity of $\mathrm{SiO}_{2}$ and $\mathrm{Al}$ in $\mathrm{BHF}$ solutions, in 2006 16th Biennial University/Government/Industry Microelectronics Symposium (2006), 225-228.

[34] G. Brambilla, F. Xu, and X. Feng, Fabrication of optical fibre nanowires and their optical and mechanical characterisation, Electron. Lett. 42 (2006) 517-519.

[35] G. Zhai and L. Tong, Roughness-induced radiation losses in optical micro or nanofibers, Opt. Express 15 (2007) 13805-13816. 